\title{
Diseño y evaluación de materiales didácticos bilingües con TICs para prácticas de laboratorio de Química
}

\author{
Beatriz Jurado-Sánchez, ${ }^{a}$ Víctor de la Asunción-Nadal, ${ }^{a}$ Laura García Carmona, ${ }^{a}$ \\ Marta Pacheco Jerez, ${ }^{\text {a }}$ Roberto María-Hormigos, ${ }^{\text {a y Tania Sierra Gómez }}{ }^{\mathrm{a}}$ \\ ${ }^{a}$ Departamento de Química Analítica, Química Física e Ingeniería Química, Universidad de Alcalá \\ (beatriz.jurado@uah.es)
}

\begin{abstract}
The new scenario of the European Higher Education Area and the increase of Erasmus student agreements has led to a rise in the number of subjects in English. All this has resulted in an increase in the participation of foreign students in undergraduate and graduate programs. There is also a growing demand on the part of the general student body to learn new subjects in English with the aim of increasing their future international employability. The use of a second language requires an adequate design of the specific didactic material without prejudice of the learning process. In this context, ICTs have been revealed as a new strategy to minimize the disadvantages listed above.

The objective of this work is the development of bilingual Spanish-English teaching material with the aim of facilitating the study and guiding the student in the learning and self-evaluation of experimental subjects in Chemistry. The new technologies will be used as PowerPoint presentations to develop an interactive laboratory notebook that will be implemented in the Moodle platform. A second objective was the evaluation of the material by biology and chemistry Erasmus bachelor's degree students.
\end{abstract}

Keywords: ICTs, practices, Chemistry, Laboratory, Erasmus, teaching competence, evaluation.

\section{Resumen}

El establecimiento del Espacio Europeo de Educación Superior y el incremento de convenios de intercambio de estudiantes Erasmus ha conducido a un aumento en la oferta de asignaturas en inglés. Todo ello ha repercutido en un aumento en la participación de estudiantes extranjeros en programas de grado y postgrado. Existe además una creciente demanda por parte del alumnado general en el aprendizaje de nuevas materias en inglés con el objetivo de incrementar su empleabilidad internacional futura. La utilización de un segundo idioma requiere un diseño adecuado del material didáctico especifico sin perjuicio del proceso realización-interpretación-aprendizaje. En este contexto, las TICs se han revelado como una nueva estrategia para minimizar las desventajas enumeradas anteriormente.

El objetivo de este trabajo es el desarrollo de material docente bilingüe español-inglés con el objetivo de facilitar el estudio y guiar al alumno en el 
aprendizaje y autoevaluación de asignaturas de experimentación en Química. Con este fin, se emplearon las nuevas tecnologías tales como presentaciones en PowerPoint para desarrollar un cuaderno interactivo de laboratorio que se implementó en la plataforma Moodle. Un segundo objetivo consistió en la evaluación del material desarrollado por parte de estudiantes internacionales Erasmus de grados en Química y Biología.

Palabras clave: TICs, prácticas, Química, laboratorio, Erasmus, competencias, evaluación.

\section{Introducción}

La Internacionalización de la Universidad Española mediante su inclusión en el Espacio Europeo de Educación Superior (EEES) ha supuesto un aumento en el número de convenios de intercambio de estudiantes Erasmus; así como una mayor participación de estudiantes extranjeros en programas de grado y postgrado. Es por ello de vital importancia asegurar la formación de los alumnos en una competencia transversal básica como es la comunicación en una segunda lengua. Existe además una demanda creciente por parte del alumnado nativo, debido a que el aprendizaje de diversas materias en inglés será cada vez más requerido para incrementar la empleabilidad internacional de los egresados de las universidades españolas.

Las prácticas de laboratorio en disciplinas científicas como la Química requieren un adecuado diseño y documentación del material didáctico para evitar que las manipulaciones a realizar consuman toda la atención y menoscaben el proceso enseñanza-aprendizaje (Hofstein \& Lunetta, 2004). La dificultad se agrava aún más cuando los contenidos se aprenden paralelamente al uso de un segundo idioma. Por ello, el material didáctico debe ofrecer información en el mayor número de modalidades sensoriales, dotando al alumnado de las competencias necesarias para realizar las técnicas propuestas, y promover la reflexión previa a la asimilación de conocimientos (Van Dyke \& Smith-Carpenter, 2017). En este contexto, las nuevas tecnologías de la información y la comunicación (TICs) se han revelado como una nueva estrategia para solventar o minimizar las desventajas enumeradas anteriormente (Palacios et al., 2016).

En esta comunicación se presentan los resultados obtenidos del proyecto de innovación docente "El empleo de la TICs para el diseño de material didáctico en la enseñanza bilingüe en el laboratorio de Química" de la Universidad de Alcalá. El presente proyecto, que fue concedido en el curso 2017-2018, ha conducido al desarrollo de material docente bilingüe español-inglés con el objetivo de facilitar el estudio y guiar al alumno en el aprendizaje y autoevaluación de asignaturas de experimentación en Química. Con este fin, se emplearon las nuevas tecnologías como presentaciones en PowerPoint y películas de video para desarrollar un cuaderno interactivo de laboratorio que se implementó en la Plataforma Moodle. El proyecto contó además con la participación de una alumna del Grado en Lenguas Modernas y Traducción de la UAH que revisó la correcta traducción de los materiales. Durante el presente curso académico la plataforma Moodle se puso a disposición de estudiantes de estudiantes internacionales de Erasmus en los grados de biología y química, 
que realizaron una encuesta acerca del uso de la misma con el objetivo de mejorar posibles deficiencias. Se espera que en un futuro los resultados obtenidos sirvan como base para una mejora futura en la práctica docente, tanto presencial como virtual.

\section{Objetivos}

El objetivo principal es el desarrollo de un cuaderno de laboratorio de Química bilingüe español-inglés. El cuaderno estará constituido por módulos o prácticas de desarrollo teórico, prácticos y de autoevaluación. Se emplearán además las nuevas tecnologías como presentaciones en PowerPoint y películas de video y el material resultante se integrará en la plataforma Moodle.

Un segundo objetivo consistirá en la preparación de una encuesta de evaluación del material desarrollado. Tanto la plataforma como la encuesta se pondrán a disposición de alumnos internacionales de Erasmus y se tomarán acciones correctivas pertinentes para su futura implementación.

Por otro lado, se pretenden alcanzar los siguientes objetivos trasversales: Facilitar el proceso enseñanza-aprendizaje en alumnos internacionales (Erasmus) que no dominen el idioma español; fomentar la formación de todos los alumnos en una competencia transversal básica: comunicación en una segunda lengua y facilitar el proceso de enseñanza-aprendizaje y el aprendizaje autónomo del alumno, potenciando la mejora de la calidad docente.

\section{Desarrollo de la innovación y resultados obtenidos}

Para el desarrollo de la innovación se plantearon dos grandes bloques metodológicos. En el desarrollo de estos participaron docentes de Química Analítica de la Universidad de Alcalá en distintas etapas profesionales: contratados FPI y FPU, contratados postdoctorales y profesores de los cuerpos docentes universitarios. Los bloques planteados se enumeran a continuación:

A. Elaboración de cuatro módulos virtuales de laboratorio atendiendo a las Guías Docentes de Operaciones Básicas de Laboratorio y Química General de los Grados en Química y Farmacia. Se abordaron las siguientes prácticas:

1. Manejo del material de laboratorio. Seguridad en el laboratorio.

2. Preparación de disoluciones.

3. Técnicas de filtración y destilación.

4. Valoraciones ácido-base.

Para cada módulo se elaboraron -en inglés y español- ficheros en PowerPoint con los fundamentos teóricos, ficheros en Word con el guion de prácticas y la resolución de problemas relacionados y cálculos estequiométricos. El material desarrollado en cada módulo se integró en la plataforma Moodle, facilitando su difusión y acceso a través de distintos dispositivos. Una alumna del Grado en Lenguas Modernas y Traducción de la Universidad 
Diseño y evaluación de materiales didácticos bilingües sobre TICs para prácticas de laboratorio de Quimica

de Alcalá (bajo la tutoría de un docente del Grado en Traducción y otra del grado en Química) revisó la correcta traducción de los materiales.

B. Elaboración y realización de encuestas de evaluación del material por parte de alumnos internacionales de Grado en Química y Biología.

Con respecto al Bloque metodológico A, se elaboraron -en inglés y español- ficheros en PowerPoint con los fundamentos teóricos, ficheros en Word con el guion de prácticas y la resolución de problemas relacionados y cálculos estequiométricos. La siguiente figura muestra un ejemplo del material docente desarrollado e integrado en la plataforma. En los mismos se han empleado distintos colores (en el ejemplo, negro para el idioma castellano y violeta para el inglés) para facilitar el aprendizaje de los alumnos extranjeros fomentando además la adquisición de competencias en ambos idiomas de todos los estudiantes.

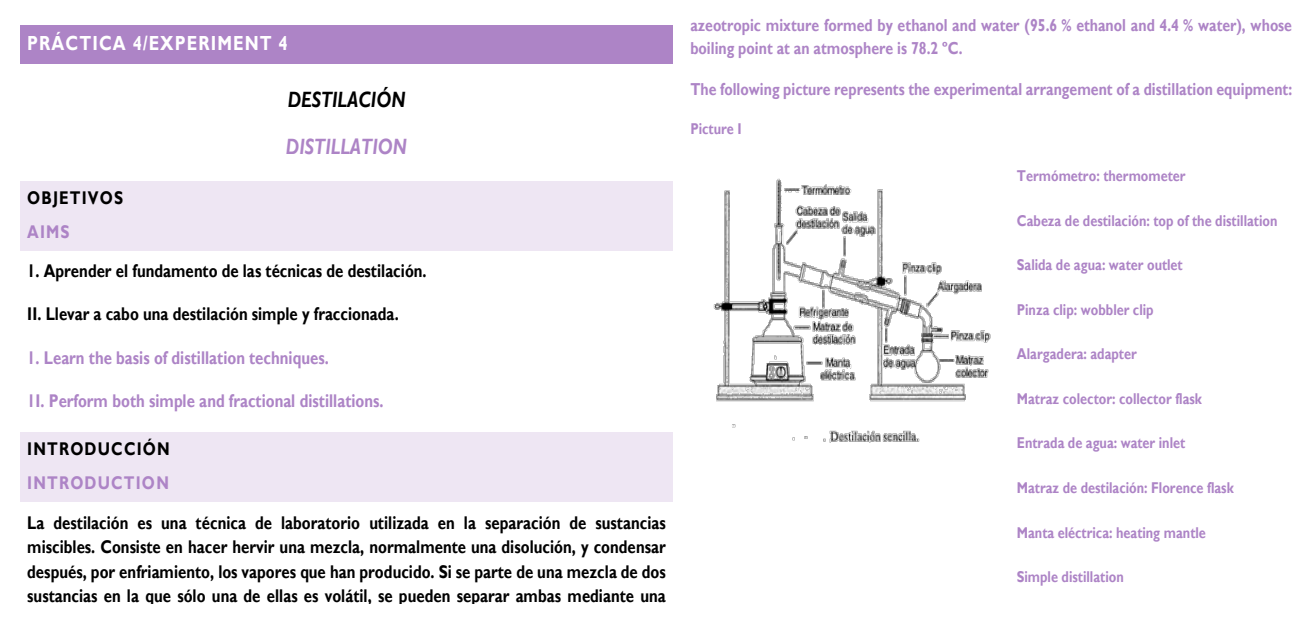

Fig.1 . Capturas de fragmentos del material docente desarrollado

Tanto los ficheros en Word como las explicaciones adicionales en PowerPoint se organizaron por módulos (cuatro en total, cada uno de ellos correspondiente a una práctica). El material está disponible en el siguiente enlace web:

https://chemistlab.moodlecloud.com/course/view.php?id=3.

Cabe destacar aquí que existen alojamientos gratuitos en la página web, que permiten el desarrollo rápido de módulos de aprendizaje y cursos.

https://moodle.com/partners $/$ ? mode $=$ search\&services $=$ hosting

La siguiente Figura muestra una captura de pantalla con la vista de la plataforma Moodle en modo alumno: 


\section{LAB PRACTICES}

Home / MY COUISES / INTRODUCTION TO COMMON LABORATORY PRACTICES FOR CHEMISTRY, BIOLOGY AND PHARMACY STUDENTS

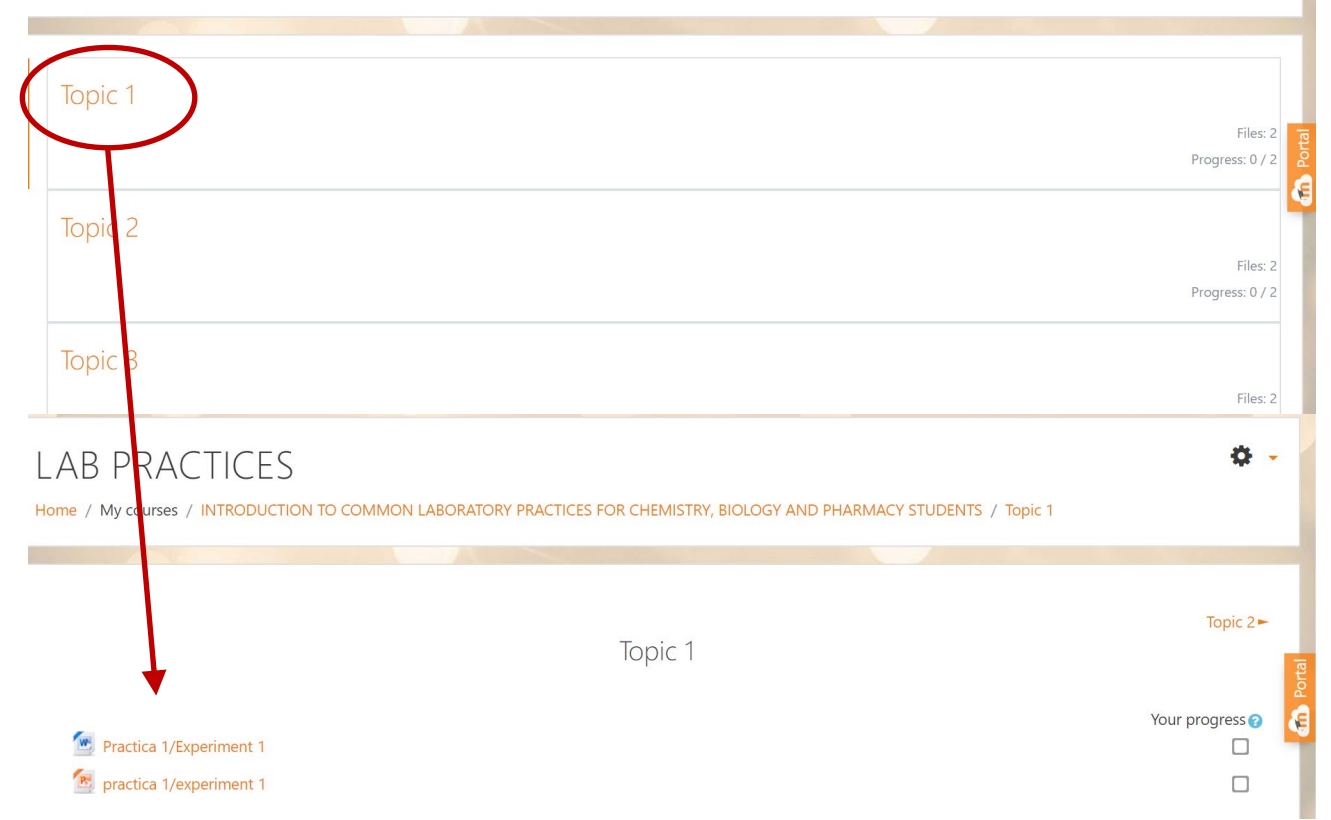

Fig.2 . Capturas de pantalla de la plataforma Moodle desarrollada y ejemplo de un módulo con el material docente integrado

Una vez finalizado el bloque anterior, se abordaron las tareas relacionadas con el bloque metodológico B. En primer lugar, se elaboró una encuesta en inglés con distintas preguntas para evaluar las debilidades y fortalezas del material desarrollado. La misma consta de seis preguntas acerca de la idoneidad de ambas traducciones para entender mejor los experimentos, la utilidad de las presentaciones de PowerPoint y la necesidad de incluir videos explicativos. También se les consultó acerca de posibles dificultades en el acceso y si ya tenían experiencia con este tipo de iniciativas, para concluir con el grado de satisfacción general. La siguiente Figura muestra la hoja de encuestas desarrollada: 
Degree:

Academic year:

1) Do you think the translation to both languages has enabled you to understand the experiment?
Totally agree
Agree
Disagree

2) Do you think that PowerPoint presentations have helped you to understand the experiment?

Totally agree

Agree

Disagree

3) Would you also like to see videos which explain the experiments beforehand?

$$
\text { Yes No }
$$

4) Did you have any problem accessing the Moodle platform?

$$
\text { Yes No }
$$

5) Have you ever used similar material in another subject?

$$
\text { Yes No }
$$

6) Indicate the percentage of usefulness you would give to these materials for a possible development of subjects related to experiments in general laboratory.

Fig.3 . Hoja de encuestas con seis preguntas para evaluar el grado de satisfacción de la plataforma Moodle.

Para realizar la evaluación se contactó con estudiantes internacionales de Italia, Francia, Reino Unido y Alemania que previamente habían visitado el laboratorio de investigación en el Departamento o cursado alguna asignatura ofertada en inglés. Se les facilitó en acceso a la Plataforma, que consultaron previamente a realizar la encuesta. Se recibieron un total de 20 encuestas, cuyos resultados se resumen a continuación: 


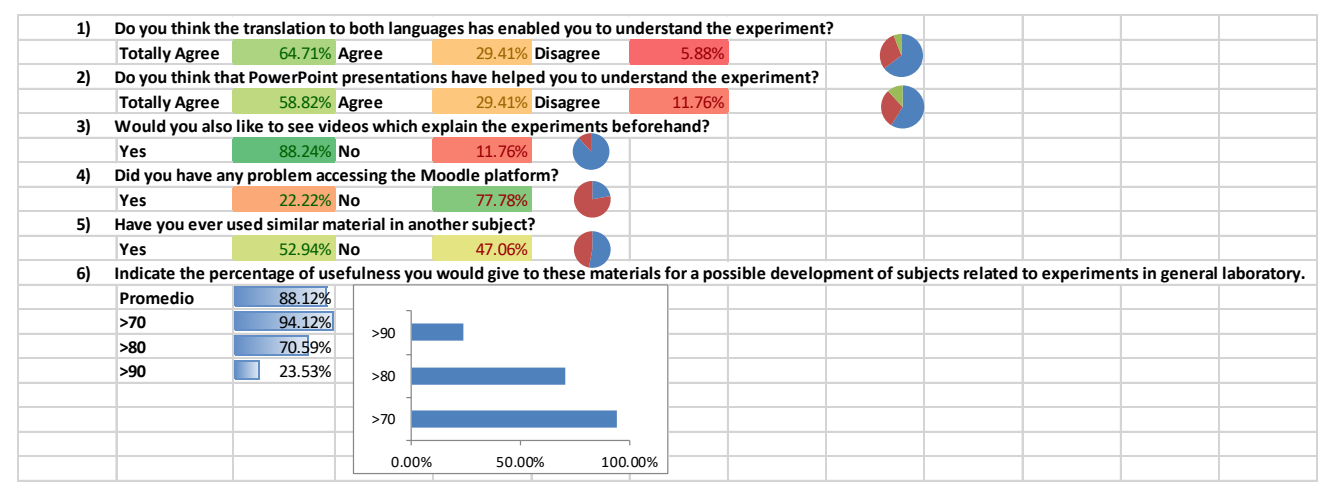

Fig.4. Resultados de la encuesta de evaluación de la Plataforma Moodle y el material docente integrado por parte de alumnos Erasmus extranjeros.

El $65 \%$ de los encuestados valoró muy positivamente la traducción simultánea a ambos idiomas (español e inglés) como herramienta para la mejora de la comprensión del experimento. Un $58 \%$ también valoró muy positivamente la utilidad de los materiales adicionales como las presentaciones de PowerPoint en este entendimiento. Como propuesta de mejora, hubo una alta demanda $(90 \%)$ y recomendación para la inclusión de videos explicativos con el desarrollo de las experiencias. Casi la mitad de los encuestados ya tenía experiencia previa en este tipo de plataformas. La valoración general final fue muy positiva, dado que el grado de utilidad estimado de la plataforma por parte de los encuestados fue de un $88 \%$.

\section{Conclusiones}

La incorporación del uso de las tecnologías de la información en la docencia universitaria supone un recurso docente que cobra especial relevancia en el aprendizaje de materias prácticas, en especial en la enseñanza en un segundo idioma como el inglés. Se ha presentado el desarrollo de material docente de prácticas generales de química tanto en inglés como en español y se ha integrado en una plataforma de enseñanza universal. Para evaluar la idoneidad y efectividad del material este se ha puesto a disposición de estudiantes internacionales y se ha realizado una encuesta de valoración. La evaluación ha sido muy positiva, con un grado de utilidad estimado de la plataforma del $88 \%$.

Se espera que el material desarrollado fomente la participación del alumnado y facilite el desarrollo de experiencias prácticas, si bien es necesario implementar y llevar a cabo su evaluación en asignaturas ofertadas en inglés (donde convergen alumnos de Erasmus y alumnos de grado que quieren complementar su formación en un segundo idioma) en futuros cursos.

\section{Referencias}

Hofstein, A. y Lunetta, VN. (2004). "The laboratory in science education: Foundation for the 21 st century" en Science Education, 88, 28-54.

Palacios, J. M., González, M. M., Bellido, D., Hidalgo, J., Naranjo, I., y Cubillana, L. (2016). "Desarrollo y aplicación de materiales virtuales en español y en inglés para un curso de 
Diseño y evaluación de materiales didácticos bilingües sobre TICs para prácticas de laboratorio de Química

Química Analítica" en International Journal of Educational Research and Innovation (IJERI), 5, 55-71.

Van Dyke, AR. y Smith-Carpenter, J. (2017). "Bring your own device: a digital notebook for undergraduate biochemistry laboratory using a free, cross-platform application" en Journal of Chemical Education, 94, 656-661.

\section{Agradecimientos}

Los autores agradecen a la Universidad de Alcalá por la concesión de los proyectos UAH/EV912 y UAH/EV1076. 